\title{
Editorial
}

\section{Engineered Nanoparticles and Their Applications}

\author{
Themis Matsoukas, ${ }^{1}$ Tapan Desai, ${ }^{2}$ and Kangtaek Lee ${ }^{3}$ \\ ${ }^{1}$ Department of Chemical Engineering, The Pennsylvania State University, University Park, PA 16802, USA \\ ${ }^{2}$ JLC Electromet, Jaipur 302013, India \\ ${ }^{3}$ Department of Chemical and Biomolecular Engineering, Yonsei University, Seoul 03722, Republic of Korea
}

Correspondence should be addressed to Themis Matsoukas; matsoukas@engr.psu.edu

Received 2 November 2015; Accepted 2 November 2015

Copyright (C) 2015 Themis Matsoukas et al. This is an open access article distributed under the Creative Commons Attribution License, which permits unrestricted use, distribution, and reproduction in any medium, provided the original work is properly cited.

Nanoparticles engineered for shape, size, and surface properties impart special functionalities including catalytic behavior, improved strength, enhanced thermal and electrical conductivity, and controlled release of host molecules. These advances have opened up applications in biomedicine, nanoenergetic materials, and functional nanocomposites. This special issue highlights successes in developing nanoparticles for a number of diverse applications.

A field in which nanoparticles are poised to play a significant role is biomedicine. Y. Shi and coworkers review recent advances in the use of FePt nanoparticles in the medical field. These alloy nanoparticles exhibit enhanced magnetic properties while also being chemically stable against oxidation. In addition to physical methods of preparation, which are scalable but suffer from poor control of morphology and size, several chemical methods have been shown to successfully produce monodisperse particles in the low nanometer range. These functionalizable and biocompatible particles find uses as MRI agents, fluorescence image probes, and targeted drug delivery, among others. This is clearly an area where much progress has been made and it is likely that in the near future FePt nanoparticles will make the transition from laboratory to clinical applications. One particularly promising biomedical application is targeted delivery. Drug losses in the gastrointestinal track are minimized and the half-life of the drug increases and allows release in a sustained manner. Thus higher bioavailability is possible with lower dosage and lower toxic side effects. K. Matlhola and coworkers study the targeted delivery of tenofovir, one of the first line drugs against HIV. The carrier particle in this study is Eudragit RS PO, a synthetic polymer that is widely used in tablets and capsules to retard the release of the drug and allow delivery in a sustained manner. Size, encapsulation efficiency, and release profile depend on several processing parameters including amount of polymer, concentration of surfactant, amount of drug, stirring speed and time, and temperature. The authors used Box-Behnken Design (BBD) to optimize the drug formulation with respect to these process parameters. The size of the particles was found to depend strongly on the polymer-to-drug ratio, surfactant concentration, and sonication time. The optimized formulation that resulted from the statistical design was characterized by very high encapsulation efficiency (98\%), good particle stability, as indicated by its zeta potential, and a sustained release profile of the drug over a period of 80 hours.

Gold nanoparticles ( $\mathrm{nAu}$ ) have been used in biomedical research quite extensively but one issue is colloidal stability in the presence of salts at physiological pH. K.-H. Tseng and coworkers studied the stability of $\mathrm{nAu}$ produced by pulse spark discharge. The authors report that carboxymethyl cellulose and polyvinyl pyrrolidone are quite effective in stabilizing nAu. Sensing of biological molecules is another area of application for gold nanostructures. R. Chauhan and T. Basu report on a highly sensitive sensor for aflatoxin B1, a toxin and potent carcinogen that is of major concern in the food processing industry. The authors describe a successful implementation of a piezoelectric sensor based on a gold coated iron oxide core-shell nanostructure functionalized to immobilize the toxin and sensed via a piezoelectric mechanism. An overlooked aspect of $n A u$ is the potential environmental impacts and toxic effects to aquatic organisms when nanoparticles are released to the environment. T. L. 
Botha and coworkers used species sensitivity distribution (SSD) to address this question. SSD is a statistical tool routinely used in assessing environmental risks by quantifying the sensitivity of aquatic species to a particular stressor. The toxicity of Au nanoparticles was examined with respect to concentration, size distribution, surface charge, surface functionality, and level of agglomeration. The toxicity of gold nanoparticles was found to be generally low. Only one species (T. sparrmanii) displayed a maximum mortality of $48 \%$ after $96 \mathrm{~h}$. Nanoparticles that are stabilized by nitrate capping lose their stability when diluted in aquatic environments and this leads to aggregation. The size of the aggregates depends on $\mathrm{pH}$ and the presence of other ionic species, but, generally, larger aggregates are more difficult to be taken up by organisms. This highlights an intricate interplay between colloidal chemistry and toxicity.

Nanoparticles and nanocomposites find new applications in sustainable energy research. J. Lui and coworkers discuss the synthesis of $\mathrm{Cu}_{2} \mathrm{Sn}_{3} \mathrm{~S}_{7}$ as the light absorbing layer for thin-film solar cells. This material, produced by the powder, baking, sulfur, and sintering (PBSS) process, exhibits an improved energy band gap and increases carrier concentration and mobility. C.-W. Ma and coworkers show how $\mathrm{ZnO}$ needle-shaped nanoparticles may be used to fabricate dyesensitized solar cells. In this example, $\mathrm{ZnO}$ produced by a chemical solution method was deposited on a fluorine-doped tin oxide (FTO) glass to form the working electrode, against a platinized FTO glass as the counter electrode. As the authors show, this system yielded a high power conversion efficiency and enhanced electron transport. $\mathrm{H}$. $\mathrm{Xu}$ and coworkers discuss the fabrication of a core-shell structured $\mathrm{Co}_{3} \mathrm{O}_{4}$-PANI nanocomposite to produce a low cost but highly efficient nanomaterial for microwave absorption. In an interesting application, G. Hernández-Padrón and coworkers produced antifog coatings from recycled polystyrene. They used silica particles to produce a nanocomposite material with hydrophobic properties and variable optical transparency.

A final application discussed in this issue is the use of nanoparticles to enhance the electric and thermal properties of colloidal suspensions. J. Li and coworkers prepared $\mathrm{Fe}_{3} \mathrm{O}_{4}$ particles with an oleate coating which were then suspended in oil to produce a highly stable nanofluid with improved dielectric breakdown behavior. Both the electric and thermal properties of suspensions are influenced very strongly by the presence of nanoparticles. S. Lotfizadeh and T. Matsoukas demonstrate these effects in a numerical study of thermal conductivity in suspensions containing colloidal clusters of various structures and shapes. The authors show that chain aggregates and nonspherical particles in general enhance the thermal conductivity.

The collection of studies in this special issue highlights the importance of engineered nanoparticles and points to multiple areas in which nanoparticle research has the potential to make new contributions.

Themis Matsoukas Tapan Desai Kangtaek Lee 

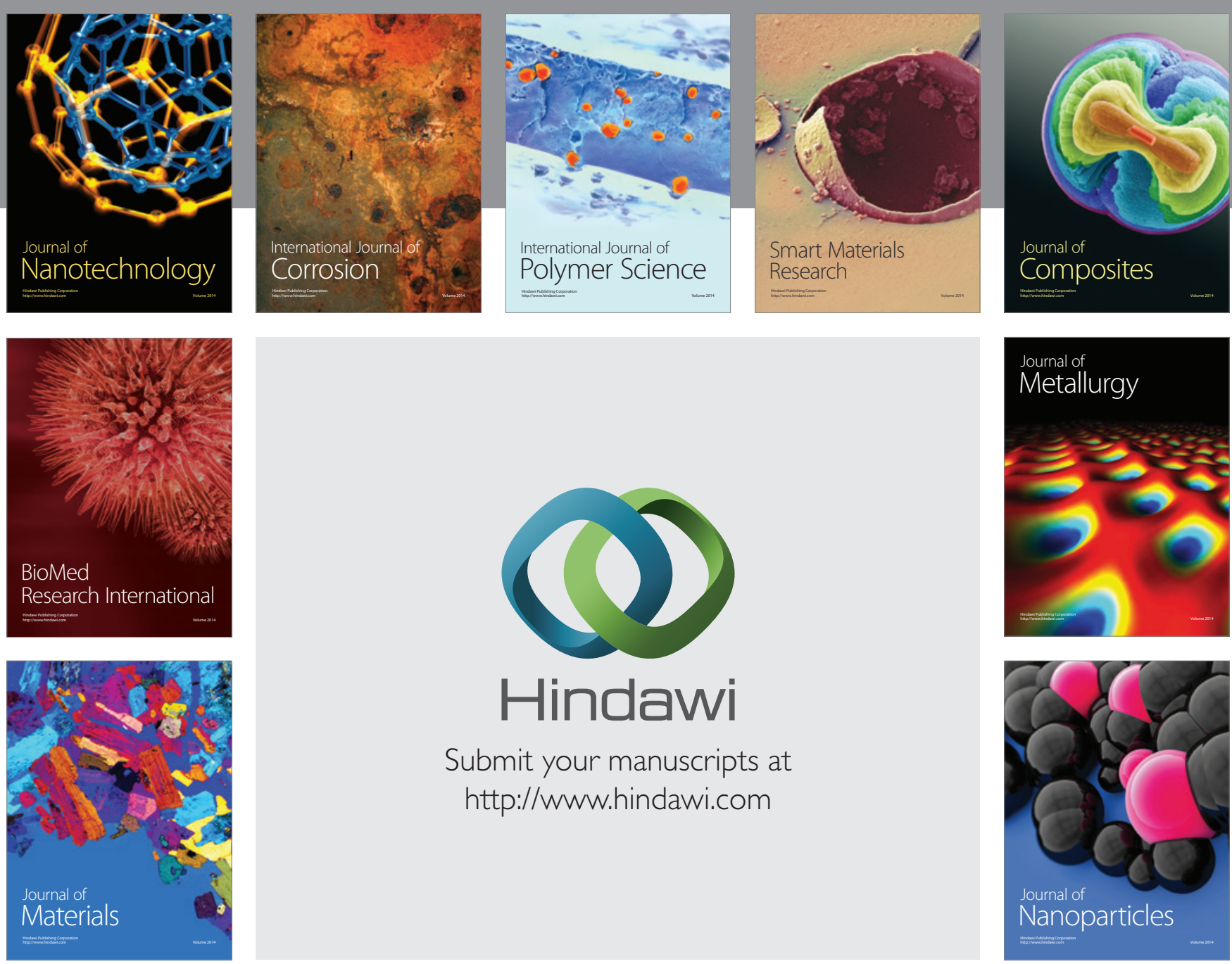

Submit your manuscripts at http://www.hindawi.com
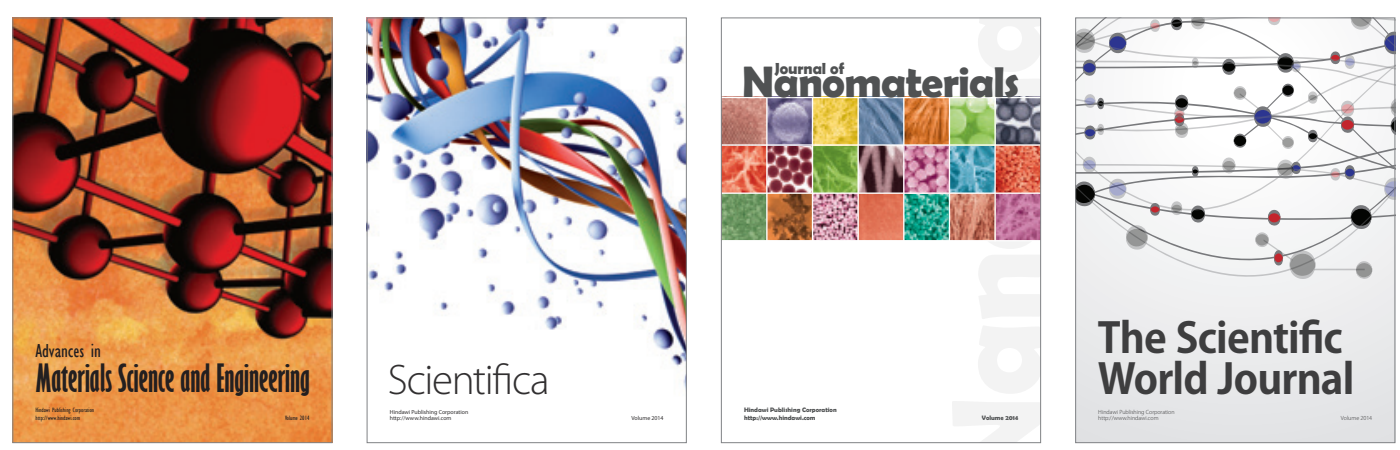

\section{The Scientific World Journal}
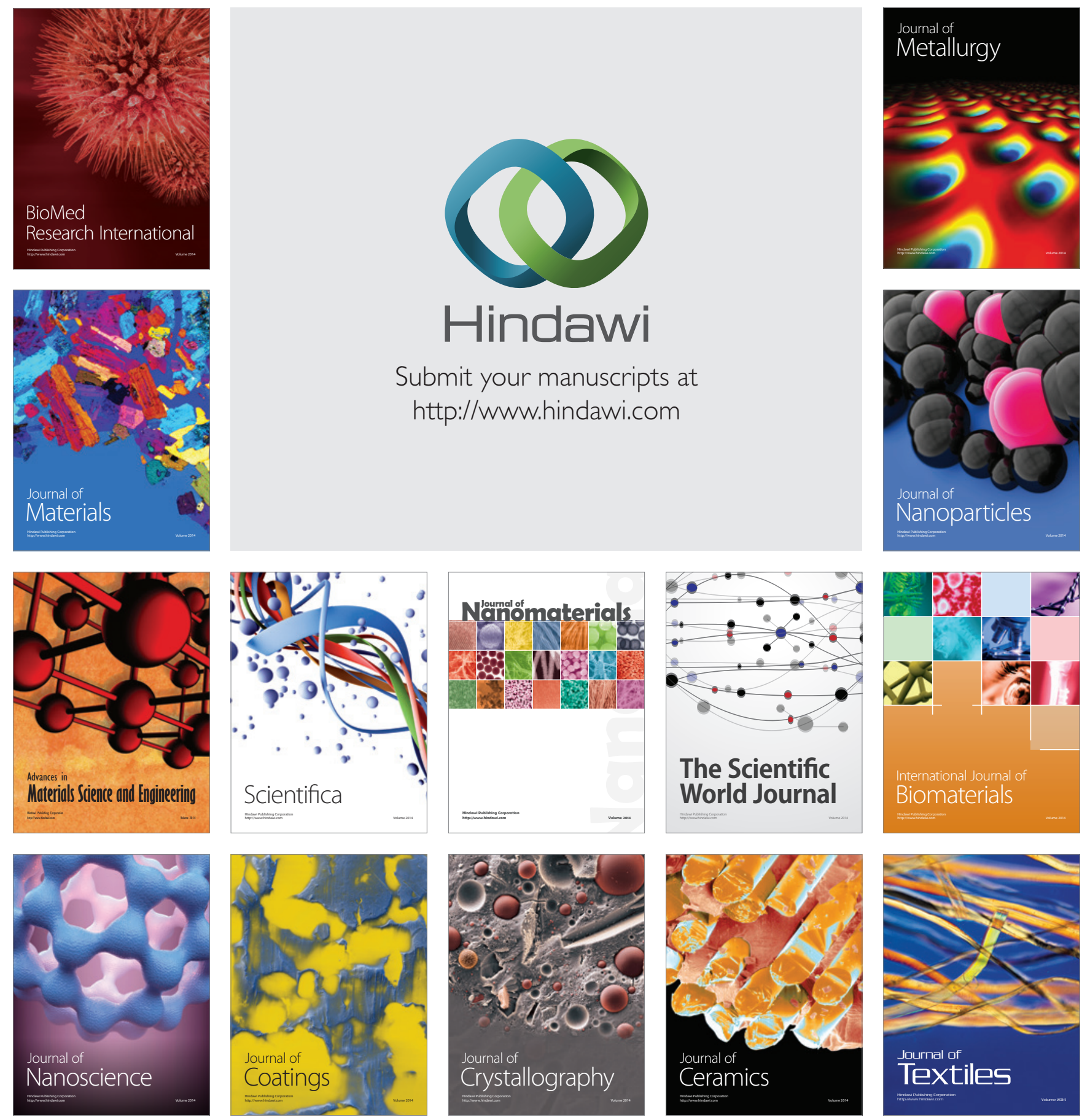